\title{
ASPECTOS DEL ORDENAMIENTO JURÍDICO EN ESPAÑA DE LA INSTITUCIÓN ÁREA DE CONOCIMIENTO DE TEORÍA DE LA LITERATURA Y LITERATURA COMPARADA
}

Antonio CHICHARRO

Universidad de Granada achichar@ugr.es

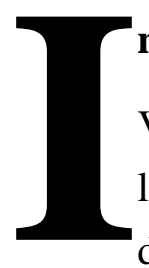

ntroducción

Voy a ocuparme del ordenamiento jurídico que ha ido configurando la institución que hoy llamamos Área de Teoría de la Literatura y Literatura Comparada en España. Trazar el rastro de la legislación, mayor y menor, existente al respecto supone el rechazo de todo afán de originalidad y, en consecuencia, la aceptación paralela de operar con instrumentos legales a los que el área de Teoría de la Literatura y Literatura Comparada debe, más que su existencia, su institucionalización y en buena medida su importante desarrollo de las últimas décadas.

El Área de Teoría de la Literatura y Literatura Comparada constituye una institución que aúna tanto lo académico y tangible como las más diversas prácticas que, intangibles y sin regulación, la nutren, producen y reproducen. Profesorado y demás investigadores, alumnado y otros usuarios venimos a ser a la postre los engarces de una cierta clase de realidad que por el simple hecho de su existencia y mantenimiento revela su necesidad e interés sociales.

Por otra parte, no cabe trazar, por no ser objeto de estas páginas, un panorama histórico de la institución Área de Teoría de la Literatura y Literatura Comparada, cuya existencia reglada entre nosotros alcanza la edad exacta de treinta y tres años ${ }^{1}$, panorama que arrancaría en todo caso muchos siglos atrás en lo que, en el solar conocido hoy como Europa, supuso la enseñanza y construcción disciplinar de la retórica, la gramática y la poética primero entre los griegos y luego entre los romanos.

\footnotetext{
${ }^{1}$ Con anterioridad, se fueron creando asignaturas y cátedras que constituyen antecedentes de las actualmente existentes y que son propias del área en cuestión. Así, si se lee el repertorio de legislación universitaria de Manuel Martínez Neira, José $\mathrm{M}^{\mathrm{a}}$ Puyol Montero y Carolina Rodríguez López (2004) podrá comprobarse que ya una Real Orden de 20 de diciembre de 1900 dispone que «se anuncien a oposición las cátedras de Teoría de la literatura y de las artes de la Facultad de Filosofía y Letras por ser de nueva creación» (Martínez Neira, Puyol Montero y Rodríguez López, 2004: 389). Con anterioridad, otra Real Orden del 12 de mayo de 1890, dispone que la asignatura de Literatura general, entre otras, sea válida como estudio en las facultades de Filosofía y Letras y Derecho (Martínez Neira, Puyol Montero y Rodríguez López, 2004: 389).
} 
Dejo a un lado, además, el consolidado Trívium altomedieval; los studia generalia en universidades y colegios medievales; así como los planes de estudio en las del humanismo renacentista; los brotes dieciochescos de informes y legislación que vienen a mostrar cierta preocupación pública por el sistema educativo dejado hasta entonces en manos sobre todo de la Iglesia católica y orientado a grupos sociales dominantes; así como el importante siglo XIX que contó en su acelerado y cambiante proceso de regulación con, por citar dos hitos importantes, el Plan Pidal ${ }^{2}$, de 1845, en el que intervino Antonio Gil de Zárate, y la Ley Moyano, de 1857, donde por cierto se indica que la primera asignatura que deben impartir las facultades de Filosofía y Letras sea la de Literatura general ${ }^{3}$, ley esta vigente hasta 1970; y obvio el tratamiento del caudal posterior que aporta el siglo XX, con sus bienios republicanos, la larga etapa del franquismo con su Ley de 19 de julio de 1943, de Ordenación Universitaria, y el así llamado periodo de la Transición que, para las universidades, se prolongó algo más que en el caso de otras instituciones españolas. Vengo a situarme entonces en el día 1 de septiembre de 1983 en que el Boletín Oficial del Estado publicaba la llamada LRU ${ }^{4}$, casi ocho años después del fallecimiento del dictador Francisco Franco.

\section{Legislación sobre las áreas de conocimiento}

\section{1. Primera aparición del concepto de área de conocimiento en la legislación sobre universidades}

Con la Ley Orgánica 11/1983 de 25 de agosto de Reforma Universitaria (LRU), publicada por Boletín Oficial del Estado (BOE), número 209 del día 1 de septiembre, se cerraba un largo periodo de transición en la universidad española al adaptarse la misma a los principios de la Constitución de 1978. Esta ley ha estado vigente hasta la publicación de la Ley Orgánica 6/2001, de 21 de diciembre, de Universidades (LOU) ${ }^{5}$. Pues bien, en el primer punto del Art. 8 del Título Primero de dicha ley, se delimita la función de los departamentos universitarios y son asociados a un área de conocimiento en los siguientes términos:

\footnotetext{
${ }^{2}$ En el Art. $6^{\circ}$ y a propósito de la regulación de la segunda enseñanza, la de ampliación que es la que preparaba para el estudio de ciertas carreras o servía para perfeccionar los conocimientos adquiridos en la elemental y que dividía la enseñanza ya en la de Letras y Ciencias, aparece entre las asignaturas de Letras una Literatura general: «Lengua inglesa, Lengua alemana, Perfección de la lengua latina, Lengua griega, Lengua hebrea, Lengua árabe, Literatura general y, en particular, la española, Filosofía con un resumen de su historia, Economía política, Derecho político y administrativo». Véase Ministerio de la Gobernación de España [Pedro José Pidal], RD aprobando el Plan General de estudios, 17 de septiembre de 1845 .

3 Art. 33. Los estudios propios de la facultad de Filosofía y Letras son: «Literatura general, Lengua y Literatura griega, Literatura latina, Literatura de las lenguas neolatinas, Literatura de las lenguas de origen teutónico, Literatura española, Historia universal, Historia de España, Filosofía e Historia de la Filosofía. A la facultad de Filosofía y Letras corresponden también los estudios de Hebreo y Caldeo, Árabe y demás lenguas orientales, cuya enseñanza tenga por conveniente establecer el Gobierno». Ley de Instrucción Pública, 9 de septiembre de 1857. En sus secciones se ocupa de los estudios, establecimientos de enseñanza, profesorado público y gobierno y administración de la instrucción pública.

${ }^{4}$ Abreviaturas usadas en este artículo: LRU (Ley Orgánica de Reforma Universitaria, de 1983), LOU (Ley Orgánica de Universidades, de 2001), BOE (Boletín Oficial del Estado), RD (Real Decreto), OM (Orden Ministerial), Res. (Resolución), Art. (Artículo).

${ }^{5}$ Véase el libro de Antonio Embid Irujo y Fernando Gurrea Casamayor, Legislación universitaria (Madrid, Tecnos, 2008), que recoge y anota el conjunto de normas, españolas y europeas, tanto de legislación básica y orgánica universitaria Derecho universitario- como del ordenamiento jurídico de la ciencia, la investigación, la formación e incluso la propiedad intelectual.
} 
Los Departamentos son los órganos básicos encargados de organizar y desarrollar la investigación y las enseñanzas propias de su respectiva área de conocimiento en una o varias Facultades, Escuelas Técnicas Superiores, Escuelas Universitarias y, en su caso, en aquellos otros centros que se hayan creado al amparo de lo previsto en el artículo 7 de esta Ley.

En el siguiente punto del mismo artículo, el 2, se añade que «los Departamentos se constituirán por áreas de conocimiento científico, técnico o artístico, y agruparán a todos los docentes e investigadores cuyas especialidades se correspondan con tales áreas».

En el Título cuarto de la ley, relativo al estudio en la universidad, se señala en el punto 1 del Art. 31, «los cursos de doctorado tendrán como finalidad la especialización del estudiante y su formación en las técnicas de investigación, dentro de un área de conocimiento».

Finalmente y en lo que la ley prevé en relación con el profesorado, en su Título quinto, vuelve a darse relevancia a la idea de área de conocimiento al vincularla en este caso con el proceso de concurso de selección del profesorado. Las comisiones, se lee en varios puntos, deberán estar compuestas por cinco profesores del área de conocimiento a la que corresponda la plaza objeto del concurso.

Como se deduce, la LRU provee así el marco legal que habría de servir para agrupar las diferentes disciplinas universitarias, configurar comisiones de selección del profesorado y poner orden en la dispersión de cátedras existentes entonces al agruparlas por el grado de aproximación científica.

\section{2. Definición legal de área de conocimiento}

De esta manera aparece en la legislación española la idea de área de conocimiento. Ahora bien, como no queda allí legislado qué se entiende por la misma, será en el RD 1888/1984 por el que se regulaban los concursos para la provisión de plazas de los cuerpos docentes universitarios (BOE núm. 257, 26 de octubre de 1984), en aplicación de la referida ley orgánica, donde se diga qué se entiende al respecto e incluso se ofrezca un primer catálogo de las mismas. En el punto 2 del Art. 2 del RD se recoge que «a tales efectos, se entenderá por área de conocimiento aquellos campos del saber caracterizados por la homogeneidad de su objeto de conocimiento, una común tradición histórica y la existencia de comunidades de profesores e investigadores, nacionales o internacionales».

En este sentido, conviene tener en cuenta que esta definición se mantendrá en el Art. 71 de la Ley Orgánica 6/2001, de 21 de diciembre, de Universidades, publicada en el BOE núm. 307, de 24 de diciembre de 2001, donde se legisla:

Artículo 71. Áreas de conocimiento.

1. Las denominaciones de las plazas de la relación de puestos de trabajo de profesores funcionarios de cuerpos docentes universitarios corresponderán a las de las áreas de conocimiento existentes. A tales efectos, se entenderá por área de conocimiento aquellos campos del saber caracterizados por la homogeneidad de su objeto de conocimiento, una común tradición histórica y la existencia de comunidades de profesores e investigadores, nacionales o internacionales.

2. El Gobierno establecerá y, en su caso, revisará el catálogo de áreas de conocimiento, previo informe del Consejo de Coordinación Universitaria. 
Parece quedar claro que, si bien esta nueva ley orgánica, la LOU, vino a derogar en 2001 a la de 1983, conocida como LRU, hizo suya con exactitud la definición del RD 1888/1984. En otros artículos de esta ley, aquellos que se refieren al procedimiento de acceso a los cuerpos de profesores universitarios, se da relevancia a las áreas de conocimiento tanto en lo que se refiere a la composición de las comisiones como a las pruebas y sus contenidos.

\section{3. Sustitución de la denominación de «área de conocimiento» por la de «ámbito de conocimiento»}

Sin embargo en el RD 56/2005, de 21 de enero, por el que se regulan los estudios universitarios oficiales de Posgrado (BOE núm. 21, de 25 de enero de 2005) y por una sola vez en el preámbulo del decreto se lee lo siguiente:

Se introduce, en consecuencia, en el sistema universitario español, junto al título de Doctor, de larga tradición en nuestra estructura educativa ${ }^{6}$, el título oficial de Máster $^{7}$ y se regulan los estudios conducentes a la obtención de ambos. La diversidad de las universidades españolas y, dentro de ellas, de los distintos ámbitos de conocimiento aconsejan dotar a los estudios de Posgrado de la mayor flexibilidad para que, en el ámbito de su autonomía, las universidades definan y desarrollen sus estrategias y la organización de la formación especializada e investigadora.

Por su parte, la Ley Orgánica 4/2007, de 12 de abril, por la que se modifica la Ley Orgánica 6/2001, de 21 de diciembre, de Universidades (LOU) (BOE núm. 89, de 13 de abril de 2007), ley que vino a cambiar el sistema de habilitación por el de acreditación y a sancionar las enseñanzas oficiales de grados, másteres y doctorado, tal como se había regulado por el RD 55/2005, de 21 enero y en el RD 56/2005 anteriormente referido (BOE núm. 21 de 25 enero 2005), además de tratar de armonizar el sistema universitario español con el del Espacio Europeo de Educación Superior (EEES), aparece un cambio en la denominación de área de de conocimiento a propósito del artículo sobre departamentos:

Artículo 9. Departamentos.

1. Los departamentos son las unidades de docencia e investigación encargadas de coordinar las enseñanzas de uno o varios ámbitos ${ }^{8}$ del conocimiento en uno o varios centros, de acuerdo con la programación docente de la universidad, de apoyar las actividades e iniciativas docentes e investigadoras del profesorado, y de ejercer aquellas otras funciones que sean determinadas por los estatutos.

Artículo 38. Doctorado.

Los estudios de doctorado, conducentes a la obtención del correspondiente título de carácter oficial y validez en todo el territorio nacional, que tienen como finalidad la especialización del estudiante en su formación investigadora dentro de un ámbito del conocimiento científico, técnico, humanístico o artístico, se organizarán y realizarán en la forma que determinen los estatutos, de acuerdo con los criterios que para la obtención del título de Doctor apruebe el Gobierno, previo informe del Consejo de Universidades. En todo

\footnotetext{
${ }^{6}$ Téngase en cuenta en este sentido el panorama histórico que a este respecto traza Aurora Miguel Alonso en su estudio «Los estudios de doctorado y el inicio de la tesis doctoral en España. 1847-1900» (2003).

${ }^{7}$ En la legislación española se consolida el anglicismo 'máster' en detrimento de 'maestría', palabra que se utiliza en el sistema universitario de no pocos países hispanoamericanos para nombrar esa clase de estudios de posgrado.

${ }^{8}$ Según el diccionario de la Real Academia Española, ‘ámbito' significa en su tercera acepción, «Espacio ideal configurado por las cuestiones y los problemas de una o varias actividades o disciplinas relacionadas entre sí». En este sentido, no creo equivocarme mucho si afirmo que la sustitución por parte del legislador de la palabra 'área' por la de 'ámbito' va en detrimento del reconocimiento de una especificidad disciplinar reguladora tanto de la actividad investigadora como la derivada de la administración de esa actividad (plazas, materias docentes, líneas de investigación, proyectos, etc.), al tiempo que da amparo a un empleo en clave de eficiencia económica, puede suponerse, antes que de rentabilidad docente y científica de los recursos humanos y materiales tanto disponibles como futuros.
} 
Aspectos del ordenamiento jurídico en España de la institución área de conocimiento...

caso, estos criterios incluirán la superación de un período de formación y la elaboración, presentación y aprobación de un trabajo original de investigación.

Llama la atención que esta denominación no vuelve a aparecer en dicha ley ni siquiera en los artículos relativos al acceso del profesorado a los cuerpos docentes.

\section{Proceso de institucionalización legal del área de conocimiento Teoría de la Literatura y Literatura Comparada (1983-2017)}

2. 1. Presencia de Teoría de la Literatura en el catálogo de áreas de conocimiento y ulterior cambio de denominación a la actual de Teoría de la Literatura y Literatura Comparada

\section{1. 1. Primer catálogo (1984)}

Por lo que concierne al catálogo de áreas de conocimiento al que se refiere el punto 2 del Art. 71 de la Ley Orgánica de Universidades de 2001, antes, en 1984, el Ministerio de Educación y Ciencia, en el ya citado RD 1888/1984 por el que se regulaban los concursos para la provisión de plazas de los cuerpos docentes universitarios, ofrecía en su anexo un primer listado con objeto de suprimir el complicado mecanismo de equiparaciones y analogías agrupando las plazas por áreas de conocimiento, lo que venía a eliminar, según el RD, denominaciones impropias, otorgando mayor versatilidad al profesorado y permitiendo a las universidades una utilización más eficiente de la plantilla. Este mismo RD preveía en su Disposición adicional segunda que cada cinco años se procedería a una revisión del catálogo de áreas y «para ello se tendrá en cuenta los avances del conocimiento científico, técnico o artístico en general y su repercusión y necesidad social en España, con objeto de suprimir o incorporar áreas y, especialmente, las que hayan podido crear las Universidades a los únicos efectos de constitución de Departamentos».

Pues bien, en este decreto aparece ya reconocida el área de Teoría de la Literatura con una descripción de las materias y correspondientes cátedras que absorbía en su denominación:

\section{TEORÍA DE LA LITERATURA}

1. «Crítica literaria» (Facultad de Filología y Facultad de Filosofía y Letras).

2. «Gramática General y Crítica literaria» (Facultad de Filología y Facultad de Filosofía y Letras).

La consecuencias que se derivaron fueron, entre otras, las siguientes: se abrió un proceso de adscripción del profesorado a las distintas áreas de conocimiento en la universidad española; se convocaron los primeros concursos conforme a lo regulado por el RD 1888/1884 con el perfil específico del área de conocimiento y comisiones integradas por profesorado del área; se fundó, por iniciativa de varios profesores del área, la Asociación Española de Teoría de la Literatura (ASETEL) ${ }^{9}$,

\footnotetext{
${ }^{9}$ En la web oficial http://www.asetel.org/ se ofrece información de la asociación cuyos fines estatutarios son: «1. La defensa de los intereses legítimos del Área de Conocimiento de Teoría de la Literatura y Literatura Comparada haciendo uso de todos los medios y vías legales necesarios en cada caso. En esta defensa se prestará especial atención a las asignaturas
} 
una asociación sobre todo profesional ${ }^{10}$, en cuyo primer simposio dedicado a «La Literatura en la enseñanza de las Humanidades» y celebrado en el Consejo Superior de Investigaciones Científicas (CSIC), en Madrid, los días 1 y 2 de Febrero de 1999, fueron tratadas en varias mesas redondas cuestiones teóricas, institucionales, curriculares y de dominio de estudio ciertamente cruciales para el área en aquel momento, tales como:

\section{-Perfil docente e investigador del profesorado del Área de Teoría de la} literatura.

-Lugar institucional del Área de Teoría de la literatura en la enseñanza de las Humanidades.

-Relación de la serie literaria con otras series culturales, atendiendo especialmente al papel de aquélla en la sociedad electrónica.

-Historia Literaria y Literatura Comparada en relación con la Teoría de la Literatura.

-Reivindicación de la lectura como fundamento de la actividad docente y tema de investigación.

\section{1. 2. Modificación del nombre del área de conocimiento de Teoría de la Literatura por el de Teoría de la literatura y Literatura Comparada}

Acuerdo de 3 de abril de 2000, de la Comisión Académica del Consejo de Universidades, por el que se crean nuevas áreas de conocimiento (BOE núm. 151, 24 de junio de 2000), y por el que se sustituye la actual denominación del área de conocimiento de «Teoría de la literatura», cuyo código de identificación es 795, por la nueva denominación «Teoría de la Literatura y Literatura Comparada» ${ }^{11}$, con el número 796 de identificación.

\footnotetext{
de carácter teórico-literario de los planes de estudios de las distintas especialidades y a la adscripción de las mismas a un área de conocimiento, así como a la Licenciatura en Teoría de la Literatura y Literatura Comparada. 2. Promover los estudios y la investigación en Teoría de la literatura y Literatura Comparada mediante la organización de actividades y reuniones científicas».

${ }^{10}$ A diferencia de otras asociaciones de investigadores próximas como las llamadas Sociedad Española de Literatura General y Comparada (SELGYC) -http://www.selgyc.com/index.php/es/- y Asociación Española de Semiótica (AES) http://www.semioticaes.es/-, entre otras existentes como la Asociación Andaluza de Semiótica (AAS) http://www.semioticaandaluza.org/-, fundadas en 1977 y 1983, respectivamente, ASETEL pone como condición para ser miembro de la misma la necesidad de que los candidatos -leemos en al Art. V de los Estatutos- «sean profesores e investigadores adscritos al Área de Conocimiento de Teoría de la Literatura y Literatura Comparada», aunque no cierra del todo la puerta a aquellos candidatos que sean «investigadores con una acreditada trayectoria en este ámbito académico y científico». En todo caso, se hace necesario estar vinculado a una universidad como personal docente e investigador.

${ }^{11}$ Este cambio venía gestándose desde tiempo atrás y más concretamente desde la celebración de una reunión de profesores de Teoría de la Literatura celebrada el 6 de noviembre de 1992 en la Universidad Autónoma de Barcelona. Allí los asistentes, entre los que me encontraba, debatieron sobre la relación entre Teoría de la Literatura y Literatura Comparada y acordaron instar a la Administración a que ambas disciplinas de los estudios literarios fueran unidas en una misma área de conocimiento, lo que implicaba un cambio de denominación al tiempo que se evitaba la dispersión de los estudios de Literatura comparada en el seno de otras áreas de conocimiento.
} 
Este acuerdo, en el que debieron pesar sin duda los argumentos del entonces rector de la Universidad de Santiago de Compostela y catedrático de Teoría de la Literatura, Darío Villanueva, portavoz de los intereses de ASETEL, conllevó un cambio administrativo en la denominación de la plaza ocupada por el profesorado a ella adscrito.

\section{1. 3. Segundo catálogo (2002)}

El primer catálogo habría de derogarse por el RD 774/2002, de 26 de julio, por el que se regula el sistema de habilitación nacional para el acceso a Cuerpos de Funcionarios Docentes Universitarios y el régimen de los concursos de acceso respectivos (BOE núm. 188, de 7 de agosto de 2002). En esta ocasión se recoge en el Anexo II el nuevo catálogo en el que, a los efectos de las pruebas de habilitación y concursos de acceso a Cuerpos Docentes Universitarios, se da entrada al área Teoría de la Literatura y Literatura Comparada con el número de identificación 796, como he dicho antes. Luego, esta disposición sería a su vez derogada por el RD 1313/2007, de 5 de octubre, por el que se regula el régimen de los concursos de acceso a cuerpos docentes universitarios (BOE núm. 241, de 8 de octubre de 2007). Llama la atención que en este RD sólo se recoge la denominación «ámbito de conocimiento» y «área de conocimiento» en los artículos 10 y 11 , respectivamente, en los que se trata de las Comisiones de reclamación y del reingreso de excedentes al servicio activo. En el resto de los más importantes artículos que regulan los concursos de acceso no aparecen nunca ni una ni otra.

\section{2. Creación del Título Universitario Oficial de Licenciado en Teoría de la Literatura y Literatura Comparada}

\section{2. 1. Antecedentes}

Hasta el momento en que se proyecta la creación de una nueva titulación universitaria, la de la licenciatura de segundo ciclo Teoría de la Literatura y Literatura Comparada, la responsabilidad en materias docentes del área se limitaba a la asignatura anual obligatoria de Crítica literaria en los planes de estudio de las distintas filologías impartidas en universidades españolas más algunas otras asignaturas optativas de diferente título en cada universidad, tales como Metodología del Análisis Literario, Géneros Literarios, Narratología, etcétera. En cualquier caso, la universidad española disponía de un cualificado e influyente profesorado de «Gramática General y Crítica Literaria» que, una vez creadas las áreas de conocimiento y separadas la «Gramática General» de la «Crítica Literaria», que se renombrarían como «Lingüística General» y «Teoría de la Literatura», respectivamente, facilitó la creación del nuevo título. En todo caso, quiero singularizar ese profesorado en un solo e influyente nombre, el de Fernando Lázaro Carreter. 


\section{2. Directrices generales del título}

RD 1450/1990, de 26 de octubre, por el que se establece el título universitario oficial de Licenciado en Teoría de la Literatura y Literatura Comparada y las directrices generales propias de los planes de estudios conducentes a la obtención de aquél (BOE núm. 278, de 20 de noviembre de 1990). El artículo 28 de la Ley Orgánica 11/1983, de 25 de agosto, de Reforma Universitaria (LRU), dispone que el Gobierno, a propuesta del Consejo de Universidades, establecerá los títulos de carácter oficial y validez en todo el territorio nacional, así como las directrices generales de los planes de estudios que deban cursarse para su obtención y homologación. Asimismo, por RD 1497/1987, de 27 de noviembre (BOE de 14 de diciembre), se establecieron las directrices generales comunes, que aparecen definidas en el propio RD como aquellas que son de aplicación a todos los planes de estudios conducentes a cualquier título universitario de carácter oficial. Así leemos:

Vertebrada, pues, la reforma académica a través de las previsiones contenidas en el citado RD 1497/1987, y de conformidad con lo dispuesto en el artículo $8 .^{\circ}$ del mismo, se trata ahora de establecer el título universitario oficial de Licenciado en Teoría de la Literatura y Literatura Comparada y las directrices generales propias de los planes de estudios conducentes a la obtención de aquél. La adecuación de las directrices generales propias al marco fijado por el RD 1497/1987 debe garantizar la necesaria coherencia y homogeneidad del modelo académico universitario.

En su virtud, vista la propuesta del Consejo de Universidades y a propuesta del Ministro de Educación y Ciencia, previa deliberación del Consejo de Ministros en su reunión del día 26 de octubre de 1990,

DISPONGO:

Artículo único.

Se establece el título universitario de Licenciado en Teoría de la Literatura y Literatura Comparada, que tendrá carácter oficial y validez en todo el territorio nacional, así como las correspondientes directrices generales propias de los planes de estudios que deben cursarse para su obtención y homologación y que se contienen en el anexo.

\section{ANEXO}

Directrices generales propias de los planes de estudios conducentes a la obtención del título oficial de Licenciado en Teoría de la Literatura y Literatura Comparada

Primera.

Las enseñanzas conducentes a la obtención del título oficial de Licenciado en Teoría de la Literatura y Literatura Comparada deberán proporcionar una formación científica adecuada en los aspectos teóricos y prácticos del hecho literario, considerado en sí mismo y desde una perspectiva comparada.

Segunda.

1. Los planes de estudios que aprueben las Universidades deberán articularse como enseñanzas de sólo segundo ciclo, con una duración de dos años. Los distintos planes de estudios conducentes a la obtención del título oficial de Licenciado en Teoría de la Literatura y Literatura Comparada determinarán, en créditos, la carga lectiva global que en ningún caso podrá ser inferior a 120, ni superior al máximo de créditos que para los estudios de sólo segundo ciclo permite el RD 1497/1987.

2. De acuerdo con lo previsto en los artículos $3 .^{\circ}, 4$, y $5 .^{\circ}$ del RD 1497/1987, de 27 de noviembre, para cursar estas enseñanzas deberán cumplirse las exigencias de titulación o superación de estudios previos de primer ciclo y complementos de formación requeridos en su caso, de conformidad con la directriz cuarta.

3. La carga lectiva establecida en el plan de estudios oscilará entre veinte y treinta horas semanales, incluidas las enseñanzas prácticas. En ningún caso la carga lectiva de la enseñanza teórica superará las quince horas semanales.

Tercera.

En cuadro adjunto se relacionan las materias troncales de obligatoria inclusión en todos los planes de estudios conducentes a la obtención del título oficial de Licenciado en Teoría de la Literatura y Literatura Comparada, con una breve descripción de sus contenidos, los créditos que deben corresponder a las enseñanzas, así como la vinculación de las mismas a una o más áreas de conocimiento.

Las Universidades asignarán la docencia de las materias troncales y/o las correspondientes disciplinas o asignaturas y, en su caso, sus contenidos, a Departamentos que incluyen una o varias de las áreas de conocimiento a que las mismas quedan vinculadas según lo dispuesto en el citado cuadro adjunto. 
Aspectos del ordenamiento jurídico en España de la institución área de conocimiento...

Cuarta.

En aplicación de lo previsto en los artículos $5 .^{\circ}$ y 8. ${ }^{\circ}, 2$, del RD 1497/1987, de 27 de noviembre, por el que se establecen directrices generales comunes de los planes de estudios de los títulos universitarios de carácter oficial, por el Ministerio de Educación y Ciencia se concretarán las titulaciones y los estudios previos de primer ciclo necesarios para cursar estas enseñanzas, así como los complementos de formación que, en su caso, deban cursarse a tal efecto según los distintos supuestos.

Título de Licenciado en Teoría de la Literatura y Literatura Comparada

\section{Materias/Créditos/Áreas}

Antropología Cultural. Análisis y estudio de la historia cultural de las Sociedades, de modo especial en relación con la producción - 6 «Antropología Social». literaria oral y escrita.

Crítica Literaria. Exposición de las principales escuelas de análisis de la obra literaria. Aplicación de métodos de análisis - -12 «Teoría de la Literatura». literario a textos de diversos géneros.

Literaturas Comparadas. Estudio y análisis comparado de temas _ -12 «Filologías correspondientes» y y textos literarios. Relaciones internas y externas. $\quad-12$ «Teorías de la Literatura».

Principios y Métodos de Literatura Comparada. Bases y métodos. --8 «Teoría de la Literatura».

Teoría del Lenguaje Literario. Sistematización de los --8 «Teoría de la Literatura». procedimientos formales literarios.

Teoría y estructura de los géneros literarios. Lírica, drama, épica: teoría, historia y estructura.

Según la OM de 11 de septiembre de 1991 por la que se determinan las titulaciones y los estudios de primer ciclo y los complementos de formación para el acceso a las enseñanzas conducentes a la obtención del título oficial de Licenciado en Teoría de la Literatura y Literatura Comparada (BOE núm. 231, de 26 de septiembre de 1991), podrán cursar las enseñanzas de la licenciatura en Teoría de la Literatura y Literatura Comparada quienes hayan superado el primer ciclo de cualquier licenciatura en filología.

OM de 13 de septiembre de 1999 por la que se amplía la de 11 de septiembre de 1991, por la que se determinan las titulaciones y los estudios de primer ciclo y los complementos de formación para el acceso a las enseñanzas de segundo ciclo conducentes a la obtención del título oficial de Licenciado en Teoría de la Literatura y Literatura Comparada (BOE núm. 223, de 17 de septiembre de 1999):

Asimismo, se podrá acceder a dichas enseñanzas desde cualquier otra titulación o primer ciclo universitario cursando, de no haberlo hecho antes, los siguientes complementos de formación:

a) 8 créditos en Teoría de la Literatura y

b) 17 créditos a elegir entre:

8 créditos en Lingüística.

9 créditos en una primera Literatura Extranjera.

9 créditos en una segunda Literatura Extranjera.

9 créditos en Literatura Española. 


\section{2. 3. Título universitario oficial de Licenciado en Teoría de la Literatura y Literatura Comparada en universidades españolas}

Res. de 30 de noviembre de 1992, de la Universidad Autónoma de Barcelona, por la que se publica el plan de estudios conducente al título oficial de Licenciado en Teoría de la Literatura y Literatura Comparada (BOE núm. 24, de 28 de enero de 1993).

Res. de 12 de julio de 1994, de la Universidad de Barcelona, por la que se hace público el plan de estudios de la Licenciatura en Teoría de la Literatura y Literatura Comparada, de esta universidad (BOE núm. 208, de 31 de agosto de 1994).

Res. de 19 de octubre de 1994, de la Universidad de Valladolid, por la que se establece el plan de estudios de Licenciado en Teoría y Literatura Comparada de la Facultad de Filosofía y Letras de Valladolid (BOE núm. 264, de 4 de noviembre de 1994).

Res. de 24 de octubre de 1996, de la Universidad Complutense de Madrid, por la que se publica el plan de estudios para la obtención del título oficial de Licenciado en Teoría de la Literatura y Literatura Comparada (BOE núm. 286, de 27 de noviembre de 1996).

Res. de 12 de mayo de 1997, de la Universidad de Granada, por la que se hace público el Plan de Estudios de Licenciado en Teoría de la Literatura Comparada, de acuerdo con el RD 1450/1990, de 26 de octubre, que se impartirá en la Facultad de Filosofía y Letras de Granada, dependiente de esta Universidad (BOE núm. 134, de 5 de junio de 1997).

Res. de 30 de julio de 1999, de la Universidad de Extremadura, por la que se publica el plan de estudios para la obtención del título de Licenciado en Teoría de la Literatura y Literatura Comparada, en la Facultad de Filosofía y Letras (BOE núm. 199, de 20 de agosto de 1999).

\section{2. 4. Otras normas que afectan al Título de Licenciado en Teoría de la Literatura y Literatura Comparada}

Res. de 10 de febrero de 2016, de la Dirección General de Política Universitaria, por la que se publica el Acuerdo del Consejo de Ministros de 22 de enero de 2016, por el que se determina el nivel de correspondencia en el Marco Español de Cualificaciones para la Educación Superior del Título de Licenciado en Teoría de la Literatura y Literatura Comparada (BOE núm. 43, de 19 de febrero de 2016). Se trata de establecer la correspondencia en el MECES, marco español para promover la movilidad de la educación superior en Europa.

\section{3. Nueva ordenación de las enseñanzas universitarias oficiales: grado y posgrado}

La licenciatura en Teoría de la Literatura y Literatura Comparada, hoy extinguida, dejaría de impartirse para dar así inicio al desarrollo de los nuevos títulos de grado, lo que se debió a un RD que vino a introducir cambios sustanciales en las enseñanzas universitarias. Se trata del RD 1393/2007, de 29 de octubre, por el que se establece la ordenación de las enseñanzas universitarias oficiales (BOE núm. 260, de 30 de octubre de 2007), que vino a derogar los anteriormente citados Reales Decretos 
55/2005 y 56/2005, ambos de 21 de enero. Este RD encuentra su base legal en La Ley Orgánica 4/2007, de 12 de abril, por la que se modifica la Ley Orgánica 6/2001, de 21 de diciembre, de Universidades, que, según se lee en el preámbulo,

[...] sienta las bases precisas para realizar una profunda modernización de la Universidad española. Así, entre otras importantes novedades, el nuevo Título VI de la Ley establece una nueva estructuración de las enseñanzas y títulos universitarios oficiales que permite reorientar, con el debido sustento normativo, el proceso anteriormente citado de convergencia de nuestras enseñanzas universitarias con los principios dimanantes de la construcción del Espacio Europeo de Educación Superior.

El RD venía a dejar en manos de las universidades la iniciativa en la creación de títulos, promovía la diversificación curricular (he de apuntar que España cuenta actualmente con 9345 titulaciones oficiales según se lee en la web de la $\mathrm{ANECA}^{12}$ ), impulsaba un cambio de metodologías docentes, imponía la consecución de competencias, buscaba la mejor posibilidad de empleo de los nuevos titulados, la movilidad, el acceso, etcétera. En el Capítulo I de este RD se incluyen las disposiciones generales, el Capítulo II establece con carácter general la estructura de las enseñanzas universitarias oficiales, que se concretan en los Capítulos III, IV y V para las enseñanzas de Grado, Máster y Doctorado -a este último le dedicaré un apartado específico más adelante-, respectivamente. Por su parte, el Capítulo VI regula los procedimientos de verificación y acreditación de los títulos. En el Anexo II se incluían las materias básicas de cada rama de conocimiento. Por supuesto que aparece la rama Arte y Humanidades, pero no se incluye expresamente entre las mismas la materia Teoría de la Literatura y Literatura Comparada, aunque sí la de Lingüística, análoga a la anterior por su dimensión teórica ${ }^{13}$. Pues bien, estas fueron las incluidas: Antropología, Arte, Ética, Expresión Artística, Filosofía, Geografía, Historia, Idioma Moderno, Lengua, Lengua Clásica, Lingüística, Literatura y Sociología. En consecuencia, el área 796 había quedado subsumida en esa etiqueta general llamada «iteratura».

Lo que ocurre a continuación en la universidad española apenas si necesita contarse por ser el suelo que pisamos y hemos ayudado a construir espoleados por la ley. Aparecen nuevos grados, nuevos másteres y nuevos programas de doctorado. Las universidades se lanzaron a la carrera a programar sus enseñanzas oficiales entre la realidad, el más variado interés y el específico del conocimiento literario en busca de futuros alumnos que vinieran a asegurar así el éxito de los respectivos proyectos universitarios. Si el legislador perseguía obtener diversidad, sin duda ha conseguido ese efecto. Bastará con leer unas listas, seguramente incompletas, de titulaciones en las que está presente con distinto grado de responsabilidad y carga docente el área de conocimiento de Teoría de la Literatura y Literatura Comparada.

\footnotetext{
${ }^{12}$ Puede verse este dato en http://srv.aneca.es/ListadoTitulos/ [consultada el 14/07/2017].

${ }^{13}$ El paralelismo que se había seguido en la convocatoria de cátedras universitarias en España de nuestras materias durante décadas del siglo XX, con la creación de las de Lengua y Literatura Española, también las de Gramática General y Crítica Literaria -más adelante, como he dicho, renombradas estas últimas Lingüística General y Teoría de la Literatura, denominación más acorde con el grado de desarrollo teórico que se vivía-, cátedras que luego se escindieron en las de Lengua Española y Literatura Española, por un lado, así como en las Lingüística General y Teoría de la Literatura, por otro, quedaba roto con este RD. Se mantenían las materias de Lengua, Literatura y Lingüística, pero no así, como digo, la de Teoría de la Literatura, presente in absentia en la de Literatura.
} 
32 Tropelías. Revista de Teoría de la Literatura y Literatura Comparada, número extraordinario 2 (2017) Antonio Chicharro

\section{3. 1. Grados (Arte y Humanidades)}

Grado en Literaturas Comparadas (Universidad de Granada)

Grado en Historia del Arte (Universidad de Granada)

Grado en Musicología (Universidad de Granada)

Grado en Literatura General y Comparada (Universidad Complutense de Madrid)

Grado en Estudios Literarios (Universidad de Barcelona)

Grado en Grado en Ciencias del Lenguaje y Estudios Literarios (Universidad de

Vigo)

Grado en Artes Escénicas (Universidad de Girona)

Grado en Grado en Artes Escénicas -Interpretación (Universidad Rey Juan Carlos)

Grado en Artes Escénicas y Mediáticas (Universidad Europea de Madrid)

Grado en Inglés Estudios Lingüísticos y Literarios (Universidad de A Coruña)

Grado en Artes Escénicas (Universidad Antonio de Nebrija)

Grado en Literatura y Escritura Creativa (Universidad de Navarra)

\section{3. 2. Grados (Ciencias Sociales)}

La presencia del área Teoría de la Literatura y Literatura Comparada resulta notable en grados de la rama de Ciencias Sociales como son los de Comunicación y Comunicación Audiovisual, presentes en muchas de las universidades españolas, públicas y privadas. No son pocos los grados en los que -así titulados, con mayor uniformidad por cierto que en el caso de no pocos de los que se integran en Arte y Humanidades, tal como se ha podido leer en el apartado anterior- el área tiene responsabilidades docentes en materias propias como introducción a los estudios literarios, géneros literarios, literatura universal, literatura comparada, además de las de estirpe semiótica, narratológica, teatral y de teoría del espectáculo, entre otras ${ }^{14}$.

\section{3. 3. Másteres ${ }^{15}$}

Máster Universitario en Estudios Literarios y Teatrales (Universidad de Granada) Máster Universitario en Estudios Lingüísticos, Literarios y Culturales (Universidad de Sevilla)

Máster Universitario en Teatro y Artes Escénicas (Universidad de Vigo)

Máster Universitario en Estudios Artísticos, Literarios y de la Cultura (Universidad de Autónoma de Madrid)

\footnotetext{
${ }^{14}$ Por lo que conozco directamente del grado de Comunicación Audiovisual de la Universidad de Granada, están adscritas al área Teoría de la Literatura y Literatura Comparada las siguientes asignaturas: Cibercultura y narraciones interactivas, Teoría y análisis del espectáculo, Representación audiovisual: estereotipos sociales y marginalidad, Adaptaciones: transmedialidad y convergencia, El cine español y sus imaginarios socioculturales y, parcialmente, Gestión de la comunicación verbal en los medios.

${ }^{15}$ En este amplio listado de titulaciones universitarias oficiales de máster, puede darse el caso de que algunas ya no se impartan.
} 
Máster Universitario en Estudios Avanzados de Teatro (Universidad de La Rioja) Máster Universitario en Investigación Literaria y Teatral (Universidad de Alcalá)

Máster Universitario en Estudios Teatrales (Universidad de Autónoma de Barcelona)

Máster Universitario en Escritura Creativa (Universidad Complutense de Madrid) Máster Universitario en Literatura Comparada: Estudios Literarios y Culturales (Universidad Autónoma de Barcelona)

Máster Universitario en Escritura Creativa (Universidad de Sevilla)

Máster Universitario en Literatura General y Comparada (Universidad de Sevilla) Máster Universitario en Estudios Literarios (Universidad Complutense de Madrid) Máster Universitario en Teatro y Artes Escénicas (Universidad Complutense de Madrid)

Máster Universitario en Teoría de la Literatura y Literatura Comparada (Universidad de Barcelona)

Máster Universitario en Historia del Drama (Universidad de Alcalá)

Máster Universitario en Literatura Española y Comparada (Universidad de León)

Máster Universitario en Literatura Comparada Europea (Universidad de Murcia)

Máster Universitario en Artes Escénicas (Universidad de Murcia)

Máster Universitario en Estudios Literarios (Universidad de Alicante)

Máster Universitario en Artes Escénicas (Universidad Rey Juan Carlos)

Máster Universitario en Estudios Teóricos e Comparados da Literatura e da Cultura (Universidad de Santiago de Compostela)

Máster Universitario en Investigación en Lenguas y Literaturas (Universidad de Valencia)

Máster Universitario en Literatura Comparada y Estudios Literarios (Universidad del País Vasco)

Máster Universitario en Artes y Ciencias del Espectáculo (Universidad del País Vasco)

Máster Universitario en Estudios Teatrales (Universidad de Barcelona)

Máster Universitario en Literatura Comparada: estudios Literarios y Culturales (Universidad Autónoma de Barcelona)

Máster Universitario en Literatura Comparada I Traducció Literária (Universidad Pompeu Fabra)

Máster Universitario en Estudios Comparados de Literatura, arte y Pensamiento (Universidad Pompeu Fabra)

Máster Universitario en Creació Literária (Universidad Pompeu Fabra)

Máster Universitario en Investigación en Letras y Humanidades (Universidad de Castilla-La Mancha) 
34 | Tropelías. Revista de Teoría de la Literatura y Literatura Comparada, número extraordinario 2 (2017) Antonio Chicharro

Máster Universitario en Estudios Avanzados en Humanidades (Universidad de La Rioja)

Máster Universitario en Estudios Comparados de Literatura, Arte y Pensamiento (Universidad Pompeu Fabra)

Máster Universitario en Literatura Española e Hispanoamericana, Teoría de la Literatura y Literatura Comparada (Universidad de Salamanca)

Máster Universitario en Literatura Española y Comparada (Universidad de León)

Máster Universitario en Formación e Investigación Literaria y Teatral en el contexto europeo (Universidad de Educación Nacional a Distancia)

Máster Universitario en Teoría y Crítica de la Cultura (Universidad Carlos III)

Máster Universitario en Literatura Europea y Enseñanzas de Lenguas (Universidad de Huelva)

Máster Universitario en Artes del Espectáculo Vivo (Universidad de Sevilla)

\section{4. El proceso de regulación del doctorado y la creación de programas con presencia del área de Teoría de la Literatura y Literatura Comparada}

\section{4. 1. La renovación de los estudios de doctorado en el marco de la LRU}

Desde los años cuarenta y hasta 1985, los estudios de doctorado en España se habían venido rigiendo por la Ley de 19 de julio de1943, de Ordenación Universitaria, ley que aportaba una muy escasa regulación en este sentido. Bastaba con cursar cuatro o cinco cursos monográficos, que organizaba cada facultad, y defender la tesis doctoral para obtener así el título de doctor. También, lo que se mantuvo hasta 1954, esta ley obligaba a que la tesis fuera juzgada en la Universidad de Madrid en tanto se autorizara por decreto a cada universidad a conferir el grado de doctor que, según el Art. 21, era de su competencia.

Será a partir de RD 185/1985, de 23 de enero (BOE núm. 41, de 16 de febrero de 1985), por el que se regula el tercer ciclo de estudios universitarios, la obtención y expedición del título de doctor y otros estudios posgraduados, cuando comience a crearse un significativo número de programas de doctorado -incluso este mismo concepto que impone la necesidad de una formación específica y coherente de la que es responsable un departamento o instituto universitario- que iba a contar con una importante presencia del área de Teoría de la Literatura y Literatura Comparada, toda vez que el citado RD obligaba a programar cursos o seminarios relacionados con la metodología y formación en técnicas de investigación; también, sobre los contenidos fundamentales de su campo, además de otros relacionados con campos afines al del programa y que fueran de interés para el proyecto de tesis doctoral. En total, según regulaba el RD, debían superarse 32 créditos -era la primera vez que se aplicaba el sistema de créditos en España- y obtener la suficiencia investigadora. De esos créditos, 20 habrían de ser de docencia y los 12 restantes de investigación. No pocas universidades españolas, las 
que contaban con profesorado suficiente del área, ofrecieron programas específicos de Teoría de la Literatura y Literatura Comparada.

El RD 778/1998, de 30 de abril (BOE núm. 104, de 1 de mayo), vino a regular $-\mathrm{y}$, en consecuencia, derogó el RD 185/1985 y otros que le sucedieron- de nuevo el tercer ciclo de estudios universitarios, la obtención y expedición del título de Doctor y otros estudios de posgrado. Pretendía profundizar lo logrado por el RD anterior tras trece años de su implantación. Para lograrlo, según se lee en su preámbulo, se proponía un número mínimo de alumnos por programa y se establecían mecanismos para la planificación conjunta y la evaluación de la calidad de los programas, entre otras medidas como la de potenciar las atribuciones de las Comisiones de Doctorado, evitar la atomización de las enseñanzas, manteniendo la división en dos períodos: el de docencia y el de investigación tutelada. Trataba también de aumentar la funcionalidad del doctorado al otorgar certificados de los distintos periodos cursados $\mathrm{y}$, en concreto, un certificado-diploma acreditativo de los estudios avanzados realizados que venía a sustituir la suficiencia investigadora: «El mencionado certificadodiploma supondrá para quien lo obtenga el reconocimiento a la labor realizada en una determinada área de conocimiento, acreditará su suficiencia investigadora, y será homologable en toda la Universidad española».

Entre otras medidas, el RD reducía a uno por universidad el número de denominaciones del título de doctor, para evitar, según se lee, «que se consideren prolongaciones de los títulos de segundo ciclo, con validez profesional». Esta nueva regulación del doctorado mantuvo y aun acrecentó el número de programas específicos propios del área de Teoría de la Literatura y Literatura Comparada en las universidades españolas, muchas de las cuales también impartía por entonces las enseñanzas conducentes a la obtención de la licenciatura de segundo ciclo en Teoría de la Literatura y Literatura Comparada. Se trataba, recordémoslo, de las universidades Autónoma de Barcelona, Barcelona, Valladolid, Complutense de Madrid, Granada y Extremadura.

\section{4. 2. La renovación de los estudios de doctorado en el marco Europeo de Educación Superior}

Como se ha podido leer, el RD 56/2005, de 21 de enero, introdujo en el sistema universitario español los estudios de posgrado y, junto al consolidado título de doctor, el nuevo título oficial de máster. Su objetivo consistía en regular los estudios oficiales de posgrado en consonancia con las líneas generales emanadas del Espacio Europeo de Educación Superior (EEES). Sin embargo, muy pronto, dos años después, el RD 1393/2007 de 29 de octubre (BOE núm. 260, de 30 de octubre de 2007), vino a establecer la ordenación de las enseñanzas universitarias oficiales $\mathrm{y}$, al tiempo que dejaba de lado el concepto de programa de posgrado, indicaba en su Art. 3.1. que las «universidades impartirán enseñanzas de Grado, Máster y Doctorado conducentes a la obtención de los correspondientes títulos oficiales». Los másteres oficiales afines a los programas de doctorado venían a considerarse periodo formativo de los mismos, por lo que para cursar el periodo de investigación, superado un máster oficial, se podría solicitar la admisión en un programa de doctorado y presentar un proyecto de tesis. 
Pero el plan de renovación aún contaría, con vigencia en este momento, con el RD 99/2011, de 28 de enero (BOE núm. 35 de 10 de febrero de 2011), por el que se regulan las enseñanzas oficiales de doctorado. Este RD prevé la creación de las Escuelas de Doctorado que han de materializar las estrategias de investigación que las universidades establezcan, las comisiones académicas de los programas de doctorado, la supervisión de las actividades del doctorando y establece una más clara distinción entre el segundo ciclo, el de máster, y el tercero, el de doctorado. También expone las competencias básicas y demás capacidades y destrezas que el doctorando debe adquirir, entre otros aspectos reguladores de la tesis doctoral, de los tribunales, de la verificación, etcétera.

2. 4. 3. Programas de doctorado con estrategias investigadoras del área de Teoría de la Literatura y Literatura Comparada

Doctorado en Lenguas, textos y contextos [Línea de investigación: Lingüística aplicada y Teoría de la Literatura y Literatura Comparada] (Universidad de Granada) Doctorado en Teoría de la Literatura y Literatura Comparada (Universidad Autónoma de Barcelona)

Doctorado en Literatura Comparada y Estudios Literarios (Universidad del País Vasco)

Doctorado en Estudios Lingüísticos, Literarios y Culturales (Universidad de Barcelona)

Doctorado en Estudios Literarios (Universidad de Alicante)

Doctorado en Teoría de la Literatura y Literatura Comparada (Universidad de Santiago de Compostela)

Doctorado en Literatura y Lenguaje (Universidad de Oviedo)

Doctorado en Lenguas Modernas y Literaturas Comparadas (Universidad de Extremadura)

Doctorado en Lengua y Literatura Catalanas y Estudios Teatrales (Universidad Autónoma de Barcelona)

Doctorado en Estudios Literarios (Universidad Complutense de Madrid)

Doctorado en Filología: Estudios Lingüísticos y Literarios, Teoría y Aplicaciones (Universidad Nacional de Educación a Distancia)

Doctorado en Literatura (Universidad de Murcia)

Programa de Doctorado en Estudios de la Literatura y la Cultura (Universidad de Santiago de Compostela)

Programa de Doctorado en Lenguas Modernas: Investigación en Lingüística, Literatura, Cultura y Traducción (Universidad de Alcalá) 
Aspectos del ordenamiento jurídico en España de la institución área de conocimiento...

\section{Consideraciones finales}

Aunque haya sido por necesidad de eficiencia administrativa de regulación del acceso a los cuerpos docentes universitarios y de gestión paralela de las materias curriculares, la introducción en la legislación universitaria del concepto de área de conocimiento, cuya definición recoge esencialmente la tríada de que debe constar un campo de conocimiento de orientación científica, esto es, una problemática común presente en lo que el RD llama «tradición histórica», un objeto de conocimiento y una comunidad de investigadores, la introducción, digo, de este concepto se ha revelado extraordinariamente positiva para la dinamización y producción del conocimiento científico, técnico, humanístico y artístico en nuestro España y, en particular, en el caso del área de Teoría de la Literatura y Literatura Comparada. Además, el hecho de vincular la producción del conocimiento con la docencia universitaria, reconociendo así los resultados especificadores del saber de orientación científica como criterio articulador y conformador de plantillas de profesorado vinculadas a departamentos universitarios, ha tenido un doble efecto: cohesión, homogeneización y desarrollo de grupos de investigadores y expansión de los dominios de su estudio, propiciada tanto por el creciente número de los mismos y el enriquecimiento de la problemática como por la proyección sobre los programas curriculares.

El crecimiento cuantitativo y cualitativo, insisto, del área de Teoría de la Literatura y Literatura Comparada, esto es, el número de investigadores, currícula y desarrollo de la problemática, ha sido exponencial desde 1984. Por eso, llama la atención que en lugar de introducir las modificaciones necesarias en la administración legal de la investigación y la enseñanza universitarias manteniendo la herramienta conceptual área de conocimiento, ésta se esté viendo desfigurada, cuando no ignorada, en las normas legales posteriores con una denominación alternativa como la de ámbito de conocimiento que es fruto no tanto del reconocimiento de la interdisciplinariedad de todo conocimiento y el sistema de sus relaciones como de la necesidad de rebajar el compromiso de la administración con la universidad española por motivos económicos de eficiencia de plantilla. Si la administración propició con la LRU un importante desarrollo del conocimiento y el sistema de la ciencia en España -me atrevo a decir- casi sin proponérselo, dado que lo que la movía era la gestión de la eficiencia de plantillas entonces muy dispersas y atomizadas en los reinos de taifas de las cátedras omnipotentes con sus profesores adjuntos y personal no numerario, ahora con plena conciencia diluye su responsabilidad al respecto y hace prevalecer criterios de eficiencia económica y de resultados a corto plazo, lo que explica el exceso de legislación en los últimos años sobre universidades, el cambio en muy cortos plazos de tiempo de criterios sobre acceso a los cuerpos docentes y la ordenación y reordenación del sistema de enseñanza en grados, másteres y doctorado.

Finalmente, si he hablado del impulso centrípeto del área, no puedo dejar de referirme a la existencia de otro impulso, el centrífugo, el que lleva a explorar nuevos dominios de estudio, elaborar nuevos objetos de conocimiento y responder así al incesante movimiento de lo real literario y sus márgenes en el mundo presente, donde alcanza su sentido el proceso de mundialización vivido y el ciberespacio. 
38 Tropelías. Revista de Teoría de la Literatura y Literatura Comparada, número extraordinario 2 (2017) Antonio Chicharro

\section{Referencias bibliográficas}

EMBID Irujo, Antonio y GURREA CASAMAYOR, Fernando (2008): Legislación universitaria. Madrid, Tecnos, $11^{\text {a }}$ edición actualizada.

HTTP://WWW.BOE.ES/

HTTP://SRV.ANECA.ES/LISTADOTITULOS/

Martínez Neira, Manuel, Puyol Montero, José Ma y RodríGuez LóPez, Carolina (2004): La legislación universitaria española 1889-1939. Madrid, Instituto Antonio de Nebrija de Estudios sobre la Universidad, Universidad Carlos III y Editorial Dykinson.

Miguel Alonso, Aurora (2003): «Los estudios de doctorado y el inicio de la tesis doctoral en España. 1847-1900», en José Ramón CRUZ MundET, ed., Archivos universitarios e historia de las universidades. Madrid, Instituto Antonio de Nebrija de estudios sobre la universidad, Universidad Carlos III, pp. 197-222. 